\title{
Iterative Channel Estimation Scheme for the WLAN Systems With The Multiple-antenna Receivers
}

\author{
Zi-Wei Zheng \\ ${ }^{1}$ College of Information Science and Engineering, Ningbo University, 315211 Ningbo, P. R. China \\ ${ }^{2}$ College of Information Engineering, Dalian Maritime University, 116026 Dalian, P. R. China \\ E-mail: ziwei_zheng@yahoo.com.cn
}

Keywords: Channel estimation; wireless local area network; Orthogonal Frequency Division Multiplexing.

\begin{abstract}
Alleviate the multipath delay spread and suitable for broadband transmission efficiency, orthogonal frequency division multiplexing wireless local area network (WLAN) is widely used to assist inverse fast Fourier transform and fast Fourier transform operation domain. Orthogonal frequency division multiplexing is a blow to the broadcast channel multipath fading and high data throughput, transmission, wireless fading channel method, which is widely used to support high performance bandwidth-efficient wireless multimedia services. Several times in the transmitter and receiver antenna technology allows data transfer rate and spectrum efficiency and the use of multiple transmit antennas and multiple receive antennas through spatial processing. High-precision channel estimation scheme is very important wideband multi-carrier orthogonal frequency complex WLAN systems use multiple antenna receiver based division of labor and the overall multi-carrier orthogonal frequency multiplexing division of performance-based WLAN system is to crucial antenna to receive the symbol error rate. In this article, the iterative channel estimation scheme proposed multi-carrier orthogonal frequency division multiplexed using multiple antennas receiver-based WLAN system.
\end{abstract}

\section{Introduction}

WLAN (Wireless Local Area Network) access providing high data rate has recently been deployed and worldwide extended to support high performance bandwidth-efficient multimedia services. Several standards such as IEEE 802.11b [1] or IEEE 802.11a/g/n [2], [3], [4], [5], [6] are transforming the way we experience personal communications. There are several other standards coming up, e.g. IEEE 802.16 [7] or IEEE 802.15 [8] that will increase capacity and range of coverage for wireless access. The technique of multiple antennas at the transmitter and the receiver (MIMO) permits several fold increase in achieved data rates and spectral efficiency through spatial processing and the use of multiple transmit and receive antennas. Due to the efficiency of mitigation multipath delay spread and being well suited for the wideband transmission, orthogonal frequency division multiplexing (OFDM) is extensively used in the wireless local area network domain with the aid of inverse fast Fourier transform and fast Fourier transform operations. The robustness of the channel estimation scheme is important for the application for the broadband multi-carrier OFDM systems with the multiple-antenna receiver and the accuracy of the channel estimation is crucial to the performance of the overall multi-carrier OFDM systems with the multiple-antenna receiver in terms of the symbol error rate (SER), including the multi-carrier OFDM based WLAN systems with the multiple-antenna receiver [1], [9]. In this paper, an iterative channel estimation scheme is proposed for the OFDM based wireless local area network systems with the multiple-antenna receiver.

\section{Problem Representation}

Referencing to the IEEE 802.11a/n standard [2], [5], [6] each packet consists of a preamble and a data carrying part. The preamble consists of ten identical short symbols (SS) and two identical long symbols (LS). Each of the SS and LS consists of $N_{S S}=16$ and $N_{L S}=64$ samples, respectively. The GI1 
field represents a guard interval for the LS. It is a replicated field consisting of the last 32 samples of the LS. At the transmitter, among OFDM symbols, the cyclic prefix is added as the guard interval (GI) to avoid the inter-symbol interference (ISI) caused by the multipath fading channel. For every OFDM symbol, there are $N=N_{L S}=64$ samples per symbol, including data sample set $D_{D}$ with $N_{D}=48$ samples, pilot sample set $D_{P}$ with $N_{P}=4$ samples and null sample set $D_{N}$ with $N_{N}=12$ samples. At the multiple-antenna receiver side, automatic gain control (AGC) and signal detection are completed at some arbitrary point in time relative to the start of the preamble field. After the AGC and signal detection are completed, the timing and frequency offset estimation starts at some unknown discrete sample index $n_{i}$ of the preamble and completed before the starting point of the GI1. Two of the LSs are used for channel estimation. The data carrying part consists of a variable number of OFDM symbols. Channel information is updated during the data carrying part. The LSs are utilized for the initial channel estimation and the pilot sample set is utilized for channel phase error tracking. From the AGC, signal detection, and the detection of the null sample set, the noise variance is also estimation. All kinds of estimated information are stored in the registers. The estimated channel information is sent for frequency domain channel equalization. Considering the $a_{r}$ th receiving antenna, after completion of AGC and signal detection at some arbitrary point in time relative to the start of the preamble field, the sampled received signal $r_{a_{t}, a_{r}}\left(n+n_{i}\right)$ from the $a_{t}$ th transmitting antenna to the $a_{r}$ th receiving antenna can be formulated as

$$
r_{a_{t}, a_{r}}\left(n+n_{i}\right)=\sum_{l=0}^{N_{h}} h_{a_{t}, a_{r l}} S\left(n+n_{i}-l\right) e^{j\left(2 \pi\left(n+n_{i}\right) f_{\varepsilon}\right) / N}+\xi_{a_{t}, a_{r}}\left(n+n_{i}\right)
$$

where $h_{a_{t}, a_{r l}}$ and $S(n)$ denote the discrete-time complex channel impulse response and the transmitted signal, respectively, $N_{h}$ is the channel length, $N$ is the IFFT/FFT size, $f_{\varepsilon}$ is the frequency offset normalized by the sub-carrier spacing $f_{\Delta}$, and $\xi_{a_{r}}(n)$ indicates AWGN. $n_{i}$ and $f_{\varepsilon}$ are estimated and corrected during the period of timing and frequency offset estimation. It is assumed that the channel does not change during one OFDM symbol and that the guard interval length is longer than the channel maximum delay spread. After the removal of the guard interval and the samples are transformed to frequency domain via a $N$ point FFT, the FFT output for the $u$ th sub-carrier signal in the $m$ th OFDM symbol can be expressed as

$$
R_{a_{t}, a_{r} u, m}=S_{u, m} \cdot H_{a_{t}, a_{r} u, m}+A_{a_{t}, a_{r u, m}}
$$

where $S_{u, m}$ is the transmitted signal on the $u$ th sub-carrier, $A_{a_{t}, a_{r u, m}}$ is a white complex Gaussian noise with variance $\sigma^{2}$ from the $a_{t}$ th transmitting antenna to the $a_{r}$ th receiving antenna, $H_{a_{t}, a_{r} u, m}$ is the channel frequency response at the sub-carrier frequency $f_{u}=u f_{\Delta}$ from the $a_{t}$ th transmitting antenna to the $a_{r}$ th receiving antenna and can be written as

$$
H_{a_{t}, a_{r} u, m}=\sum_{l=0}^{N_{h}} h_{l} e^{-j 2 \pi u \tau_{l} f_{u}}
$$

Where $\left\{\tau_{l}\right\}$ are the path time delays, $f_{\Delta}$ is the sub-carrier spacing. In the $802.11 \mathrm{a} / \mathrm{n}$ protocol, after completion of AGC, signal detection, timing and frequency offset compensation, the initial channel frequency response estimation is performed based on the two identical LSs. From equation (2), to reduce the estimation variance, the initial least square channel frequency response $\hat{H}_{a_{t}, a_{r u}}{ }^{L}$ at the $u$ th sub-carrier from the $a_{t}$ th transmitting antenna to the $a_{r}$ th receiving antenna can be estimated by averaging over the two LSs as

$$
\hat{H}_{a_{t}, a_{r u}}{ }^{L}=\frac{R_{a_{t}, a_{r} u, 1}^{L}}{S_{a_{t}, a_{r} u, 1}^{L}}+\frac{R_{a_{t}, a_{r} u, 2}^{L}}{S_{a_{t}, a_{r} u, 2}^{L}}
$$


where $R_{a_{t}, a_{r u, 1}}{ }^{L}$ and $R_{a_{t}, a_{r}, 2}{ }^{L}$, respectively, denotes the FFT output for the $u$ th sub-carrier signal in the first and the second LS OFDM symbol. $S_{a_{t}, a_{r} u, 1}^{L}$ and $S_{a_{t}, a_{r}, 2}^{L}$, respectively, denotes the transmitted signal on the $u$ th sub-carrier in the first and the second LS.

Though the least square approach is simple to use, it also suffers performance degradation by additive random noise, especially at low SNR. In order to reduce the random noise effect at sub-carrier channels, the initial least square channel frequency response $\hat{H}_{a_{t}, a_{r u}}{ }^{L}$ at the $u$ th sub-carrier from the $a_{t}$ th transmitting antenna to the $a_{r}$ th receiving antenna are weighted and averaged over three neighboring sub-carrier channels under the assumption that the channel frequency response varies slowly over adjacent sub-carrier channels. The weighted and averaged channel frequency response $\hat{H}_{a_{t}, a_{r} u}{ }^{L, W}$ at the $u$ th sub-carrier from the $a_{t}$ th transmitting antenna to the $a_{r}$ th receiving antenna is

$$
\hat{H}_{a_{t}, a_{r} u}^{L, W}=\frac{1-\alpha}{2} \hat{H}_{a_{t}, a_{r}(u-1)}^{L}+\alpha \hat{H}_{a_{t}, a_{r} u}^{L}+\frac{1-\alpha}{2} \hat{H}_{a_{t}, a_{r}(u+1)}^{L}
$$

The weighting factor, $\alpha \in[0,1]$, is determined by the following rules: (a) the value of $\alpha$ needs to be increased when the channel frequency response varies fast over the adjacent sub-carrier channels; (b) the value of $\alpha$ needs to be decrease as the SNR decreases. During the $i$ th data carrying part OFDM symbol, based on the pilot sub-carriers, the least square channel frequency response $\hat{H}_{a_{t}, a_{r} k_{p}, i}^{P}$ at the $k_{p}$ th sub-carrier from the $a_{t}$ th transmitting antenna to the $a_{r}$ th receiving antenna can be expressed as

$$
\hat{H}_{a_{t}, a_{r} k_{p}, i}^{P}=\frac{R_{a_{t}, a_{r} k_{p}, i}^{P}}{S_{k_{p}, i}^{P}}
$$

where $k_{p}$ is the sub-carrier index for the pilot symbol, $R_{a_{t}, a_{r} k_{p}, i}^{P}$ denotes the FFT output for the $k_{p}$ th sub-carrier signal in the $i$ th data carrying part OFDM symbol, $S_{k_{p}, i}^{P}$ denotes the transmitted pilot signal on the $k_{p}$ th sub-carrier in the $i$ th data carrying part OFDM symbol.

Since the period of two OFDM symbols is very short, the fluctuations of the channel gains between consecutive OFDM symbols under the IEEE 802.11a/n standard defined WLAN environments can be negligible. The channel frequency response at the $k_{p}$ th sub-carrier of the first data carrying part OFDM symbol can be re-expressed as

$$
\hat{H}_{a_{t}, a_{r} k_{p}, 1}^{P}=\hat{H}_{a_{t}, a_{r} k_{p}}^{L, W} \cdot e^{-j \hat{\phi}_{a_{t}, a_{r} k_{p}, 1}^{P}}
$$

where $\hat{\phi}_{a_{t}, a_{r} k_{p}, 1}^{P}$ is the phase rotation of the estimated first data carrying part OFDM symbol channel frequency response from the estimated two LSs channel frequency response at the $k_{p}$ th sub-carrier. Assume that the phase rotation of the estimated first data carrying part OFDM symbol channel frequency response from the estimated two LSs channel frequency response at the $u$ th sub-carrier $\hat{\phi}_{a_{t}, a_{r} u, 1}$ are linearly increasing with the sub-carrier index $u$, based on and by averaging over all the pilot sub-carriers of the first data carrying part OFDM symbol, $\hat{\phi}_{a_{t}, a_{r} a_{r u, 1}}$ can easily be calculated.

Then, the channel frequency response $\hat{H}_{a_{t}, a_{r} u, 1}$ at the $u$ th sub-carrier of the first data carrying part OFDM symbol can be weighted and averaged as

$$
\widetilde{H}_{a_{t}, a_{r} u, 1}=\beta \hat{H}_{a_{t}, a_{r} u}^{L, W} \cdot e^{-j \hat{\phi}_{a_{t}, a_{r, u}, 1}}+(1-\beta) \hat{H}_{a_{t}, a_{r} u}^{L, W}
$$

The weighting factor, $\beta \in[0.5,1]$, is determined by the SNR and Doppler frequency as following rules: the value of $\beta$ needs to be increased and closed to 1 when the SNR becomes infinite and Doppler frequency is very high. The sub-carrier specific received complex value symbol $R_{a_{t}, a_{r} u, 1}$ of the first 
data carrying part OFDM is equalized by applying the simple zero forcing technique, see equation (9), based on the previously estimated transfer factor $\widetilde{H}_{a_{t}, a_{r u, 1}}$ of equation (8) that is taken as a prediction of the current transfer factor

$$
\hat{S}_{a_{t}, a_{r} u, 1}=\frac{R_{a_{t}, a_{r u, 1}}}{\widetilde{H}_{a_{t}, a_{r u, 1}}}
$$

Then, the equalized symbol $\hat{S}_{a_{t}, a_{r} u, 1}$ is detected by the demodulator. The detected symbol $\bar{S}_{a_{t}, a_{r u, 1}}$ is further divided by the received symbol $R_{a_{t}, a_{r} u, 1}$ in order to calculate the new channel transfer factor $\hat{H}_{a_{t}, a_{r} u, 1}$

$$
\hat{H}_{a_{t}, a_{r u, 1}}=\frac{R_{a_{t}, a_{r u, 1}}}{\bar{S}_{a_{t}, a_{r u, 1}}}
$$

Equation (10) is used for the estimation of the channel frequency response of all the pilot sub-carriers of the second data carrying part OFDM symbol.

Using the same method as that used for the channel frequency response $\hat{H}_{a_{t}, a_{r} u, 1}$ at the $u$ th sub-carrier of the first data carrying part OFDM symbol, the channel frequency response $\hat{H}_{a_{t}, a_{r} u,(i+1)}$ at the $u$ th sub-carrier of the $(i+1)$ th data carrying part OFDM symbol (where $i \geq 1$ ) from the $a_{t}$ th transmitting antenna to the $a_{r}$ th receiving antenna can be weighted and averaged as

$$
\widetilde{H}_{a_{t}, a_{r u},(i+1)}=\beta \hat{H}_{a_{t}, a_{r} u, i} \cdot e^{-j \hat{\phi}_{a_{t}, a_{u,},(i+1)}}+(1-\beta) \hat{H}_{a_{t}, a_{r u, i}}
$$

where $\hat{\phi}_{a_{t}, a_{r},(i+1)}$ is the phase rotation of the estimated $(i+1)$ th data carrying part OFDM symbol channel frequency response from the estimated $i$ th channel frequency response on the $u$ th sub-carrier. The weighting factor, $\beta \in[0.5,1]$, is determined by the SNR and Doppler frequency as following rules: the value of $\beta$ needs to be increased and closed to 1 when the SNR becomes infinite and Doppler frequency is very high.
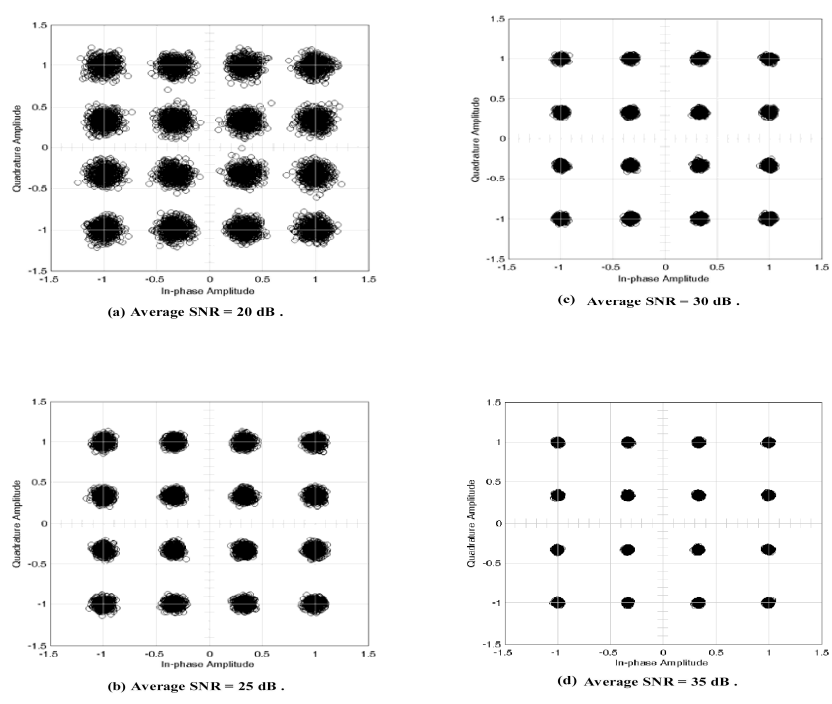

Fig. 1. Constellation of 16 QAM for the designed $2 \times 2$ wireless local area network system under Channel E with different SNR

To further improve the channel estimation performance, an iterative algorithm is developed based on the expectation-maximization (EM) algorithm [10] for each receiving antenna at the receiver. The noise variance is smoothed and estimated by using those null symbols according to the signal frame format of the multi-carrier OFDM based WLAN systems with the multiple-antenna receiver [6]. The wireless local area network system with 2 transmitting antennas and 2 receiving antennas is considered, the BRAN Channel E indicating a typical harsh fading channel model for large open space NLOS environment [4]. 16 QAM are used as the data carrying part sub-carrier modulation 
modes. The channel response zero-forcing equalized constellation points of 16 QAM signal of the designed wireless local area network system with different average SNR under BRAN Channel E is shown in figure 1. From figure 1, we can see that the proposed channel estimation scheme can make the designed wireless local area network system with practical system performance.

\section{Summary}

An iterative channel estimation scheme is proposed for the wireless local area network systems with multiple-antenna receiver. Performance evaluation shows that the wireless local area network system with multiple-antenna receiver is with practical system performance under harsh fading channel by use of the proposed channel estimation scheme.

\section{Acknowledgments}

This work was supported in part by the National Science Foundation of China (No. 60972063), the National Science and Technology Major Project (No. 2011ZX03002-004-02), the Science Foundation of Zhejiang Province (No. R1110416), the Specialized Research Fund for the Doctoral Program of Higher Education (No. 20113305110002), the Program for Technology Innovation Team of Ningbo Government (No. 2011B81002).

\section{References}

[1] "WLAN Medium Access Control (MAC) and Physical Layer (PHY) specifications: High-Speed Physical Layer extension in the 2.4 GHz band". IEEE 802.11b, 1999.

[2] "WLAN Medium Access Control (MAC) and Physical Layer (PHY) specifications: High-Speed Physical Layer in the 5 GHz Band". IEEE 802.11a. 1999.

[3] "WLAN Medium Access Control (MAC) and Physical Layer (PHY) specifications: Further Higher Data Rate Extension in the 2.4 GHz Band". IEEE 802.11g. 2003.

[4] IEEE 802.11 Task Group n, “Channel Model,” May 2004.

[5] IEEE 802.11 Task Group n, "Usage Models," rev. 19, May 2004.

[6] IEEE 802.11n-2009-Amendment 5: Enhancements for Higher Throughput. IEEE-SA. 29 Oct., 2009.

[7] "Part 16: Air Interface for Fixed Broadband Wireless Access Systems. Amendment 2: Medium Access Control Modifications and Additional Physical Layer Specifications for 2-11 GHz". IEEE 802.16a. 2003

[8] "Multiband OFDM physical Layer Proposal for IEEE 802.15 task Group 3a". IEEE 802.15.3a. 2003.

[9] C. F. Wu, M. T. Shiue and C. K. Wang, "Joint Carrier Synchronization and Equalization Algorithm for Packet-Based OFDM Systems Over the Multipath Fading Channel", IEEE Trans. Vehicul. Technology, Vol. 58, No. 9, pp. 248-260, 2009.

[10]J. Choi. Adaptive and Iterative Signal Processing in Communications. Cambridge, U.K.: Cambridge University Press, 2006. 